\title{
The role of basic laboratory parameters in diagnosing acute appendicitis and determining disease severity in the elderly
}

\author{
Mustafa Yeni ${ }^{1}$ and Rifat Peksöz ${ }^{2}$ \\ ${ }^{1}$ Erzurum City Hospital \\ ${ }^{2}$ Affiliation not available
}

April 26, 2021

\begin{abstract}
Background: Abdominal pain constitutes the most common complaint for geriatric patients who present to the emergency department, with nearly $20 \%$ suffering from acute appendicitis (AA). Although key for diagnosis, clinical symptoms tend to be weak and atypical in the elderly. Therefore, patients present late to health institutions. Hence, prognosis and complication rates are worse in the elderly. Here, we aimed to reveal the role of basic laboratory parameters in diagnosing AA and determining disease severity. Methods: 143 elderly patients who underwent appendectomy were retrospectively analyzed. The patients were divided into three groups based on AA severity as Group I: negative appendectomy ( $\mathrm{n}=15)$; Group IIa: uncomplicated appendicitis $(n=79)$; Group IIb: complicated appendicitis $(n=49)$. Results: We found no difference between the groups for age, sex, or comorbid diseases $(\mathrm{p}>0.05)$. As the time of admission to the hospital increases, the severity of the disease increases. Group IIb had higher length of stay and complication rates $(\mathrm{p}<.0 .05)$ Conclusions: Preoperative WBC, neutrophil, NLR, MPV, CRP, and direct and total bilirubin levels can be used as biomarkers to determine AA diagnosis in the elderly. NLR, PLR, RDW, CRP, and direct and total bilirubin levels can be used to determine the presence of complications in appendicitis.
\end{abstract}

The role of basic laboratory parameters in diagnosing acute appendicitis and determining disease severity $i$

*: Group IIb was significantly higher than other groups (group I, group IIa) in Post-hoc analysis

The patients were evaluated for total bilirubin, direct bilirubin, CRP and 11 hemogram subparameters. Yet, only subparameters with a high diagnostic value were discussed.

There were significant differences between the groups in terms of neutrophil, NLR, CRP, TB, and DB levels $(\mathrm{p}<0.05)$.

WBC, neutrophil, NLR, MPV, total bilirubin, direct bilirubin, and CRP levels were found as markers with high diagnostic value for diagnosing acute appendicitis (Table 2, Figure 1). 
Table 2. The ROC analysis for normal appendix and acute appendicitis

\begin{tabular}{llllll}
\hline Parameters & Cutt-off value & $\begin{array}{l}\text { AUC } \\
(\mathrm{p})\end{array}$ & $\begin{array}{l}\text { Sensitivity } \\
(\%)\end{array}$ & $\begin{array}{l}\text { Specificity } \\
(\%)\end{array}$ & $\begin{array}{l}\text { LR+ } \\
\text { CRP }\end{array}$ \\
WBC & 1,23 & $\begin{array}{l}0,668 \\
(0,143)\end{array}$ & 77 & 57 & 1,81 \\
Neutrophil & 8,97 & $\begin{array}{l}0,722 \\
(0,052)\end{array}$ & 85 & 57 & 1,98 \\
NLR & 6,36 & $\begin{array}{l}0,743 \\
(0,033)\end{array}$ & 86 & 71 & 3,02 \\
MPV & 3,28 & $\begin{array}{l}0,700 \\
(0,080)\end{array}$ & 83 & 71 & 2,93 \\
Total Bilirubin & 0,695 & $\begin{array}{l}0,624 \\
(0,277)\end{array}$ & 88 & 42 & 1,55 \\
Direct Bilirubin & 0,235 & $\begin{array}{l}0,718 \\
(0,057)\end{array}$ & 61 & 85 & 4,32 \\
& & 0,716 & 65 & 85 & 4,58 \\
\end{tabular}

Abbreviations: CRP; C-reactive protein, WBC: White blood cell, NLR; Neutrophil to lymphocyte ratio, MPV; Mean platelet volume

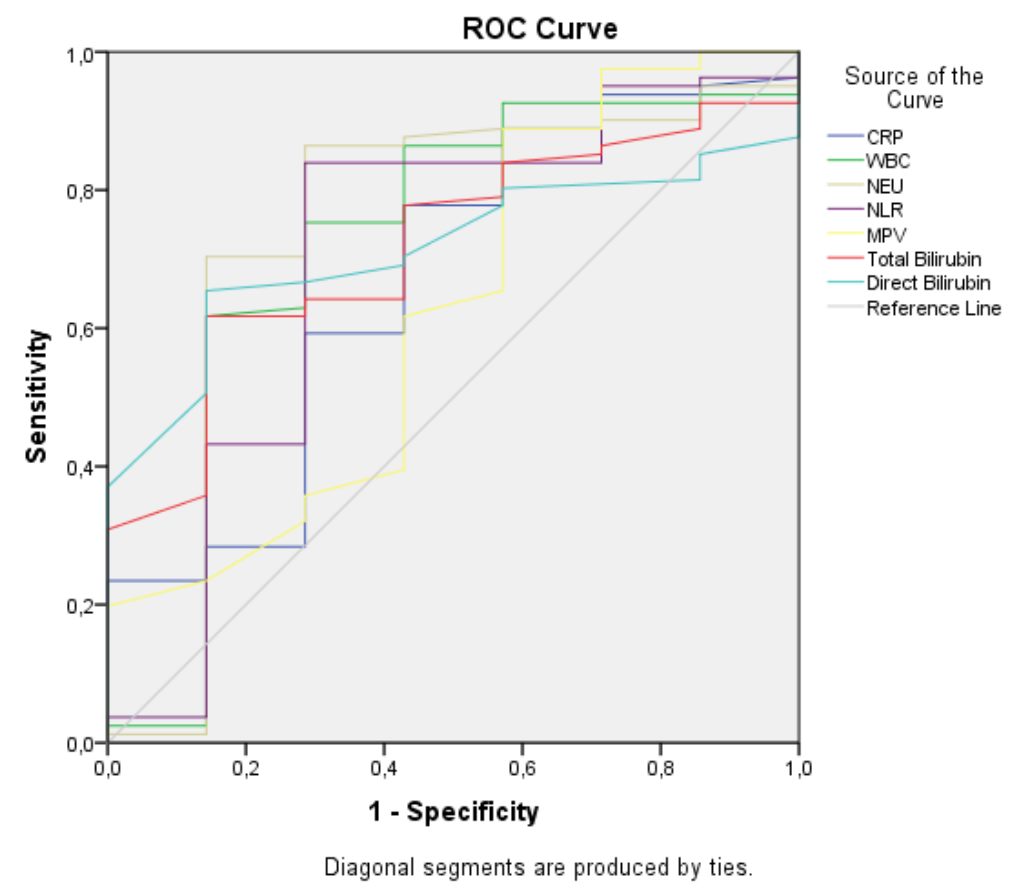

NLR, PLR, RDW, CRP, TB, and DB levels were found as markers with high diagnostic value for differentiating between complicated and uncomplicated appendicitis (Table 3, Figure 2).

Table 3. The ROC analysis for complicated and uncomplicated appendicitis 


\begin{tabular}{llllll} 
Parameters & Cutt-off value & $\begin{array}{l}\text { AUC } \\
(\mathrm{p})\end{array}$ & $\begin{array}{l}\text { Sensitivity } \\
(\%)\end{array}$ & $\begin{array}{l}\text { Specificity } \\
(\%)\end{array}$ & $\begin{array}{l}\text { LR }+ \\
\text { CRP }\end{array}$ \\
NLR & 5,11 & $\begin{array}{l}0,833 \\
(<0,000)\end{array}$ & 72 & 76 & 3,00 \\
PLR & 5,10 & $\begin{array}{l}0,617 \\
(0,065)\end{array}$ & 72 & 44 & 1,28 \\
RDW & 190,62 & $\begin{array}{l}0,641 \\
(0,027)\end{array}$ & 50 & 82 & 2,77 \\
Total Bilirubin & 0,87 & $\begin{array}{l}0,634 \\
(0,035)\end{array}$ & 69 & 60 & 1,73 \\
Direct Bilirubin & 0,30 & $\begin{array}{l}0,621 \\
(0,056)\end{array}$ & 52 & 72 & 1,88 \\
& 13,15 & 0,690 & 58 & 78 & 2,65 \\
\hline
\end{tabular}

Abbreviations: CRP; C-reactive protein, NLR; Neutrophil to lymphocyte ratio, PLR: platelet to lymphocyte ratio, RDW; red cell distribution width

Figure 2: ROC analysis for uncomplicated and complicated appendicitis

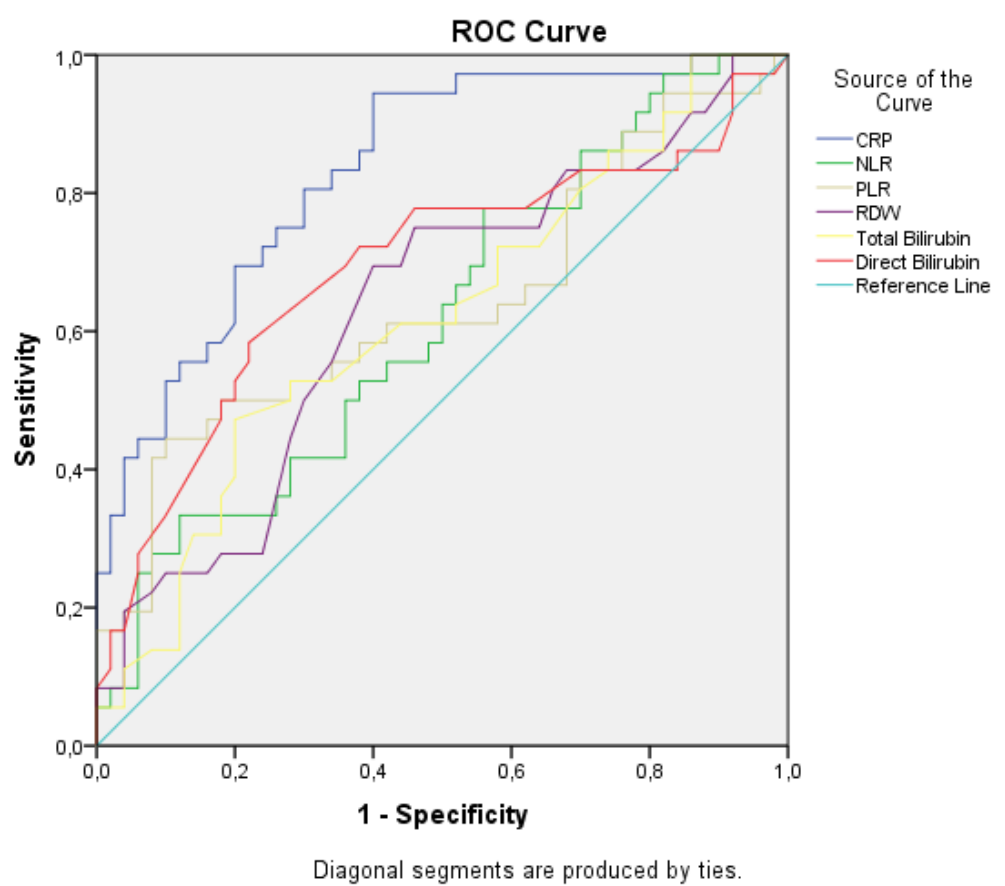

Of the patients, $2.8 \%$ were operated under spinal anesthesia and $97.2 \%$ under general anesthesia. 102 of the operations ( $71.3 \%)$ were open ( $48.3 \%$ Mc Burney, $17.5 \%$ paramedian, $5.6 \%$ midline) and $42(28.7 \%)$ were laparoscopic.

Length of hospital stay was $3.13 \pm 2.56$ days in group I, $3.34 \pm 2.01$ days in group IIa, and $6.59 \pm 5.93$ days in group IIb, with a significant difference between the groups $(\mathrm{p}<0.05)$ (Table1).

Postoperative complication rate was $35.6 \%$. Complication rates in the groups were $2.09 \%$ in group I, $16.7 \%$ in group IIa, and $16.7 \%$ in group IIb, with significant differences $(\mathrm{p}<0.05)$ (Table 1$)$. No mortality was 
observed.

\section{DISCUSSION}

In elderly individuals, rebound sensitivity decreases due to atrophy of the abdominal muscles, along with increased pain threshold due to conduction differences in the nervous system and certain changes in the detection and limitation of pain. Hence, the clinical picture tends to be atypical and obscure in the elderly [11]. The time from the onset of symptoms to hospital admission and surgery also seems higher in the elderly $[12,13]$. Delayed admission leads to perforation of the appendix. Acute appendicitis perforation is observed in 18-34\% of the general population [6]. However, this rate increases up to $41-56.3 \%$ in geriatric patients $[2,3,9]$. Male sex, anorexia, fever $[?] 38^{[?]} \mathrm{C}$, and duration of pain before admission are risk factors for perforated appendicitis. The most important factor remains delayed admission to hospital [3,6]. Male patients are observed to be more reluctant for admission to hospital and therefore present later [14]. In the current study, the rate of complicated appendicitis was found as $34.27 \%$, somewhat below the data in the literature. Time to hospital admission remains the most important factor for perforation, with a mean of 3.3 days for complicated appendicitis patient group. Gender plays no role as a factor in the occurrence of complicated appendicitis.

Comorbid diseases tend to increase morbidity and mortality, although they have not been identified as a significant factor for perforation [3]. For elderly patients, the rate of comorbid disease is observed to be 43$60.7 \%[3,6]$. The rate of comorbid disease in our sample was higher than the reports in the literature (69.9\%), with no significant difference between the groups, suggesting that it is not a risk factor for complicated appendicitis.

There is no clinical symptom, finding, laboratory test, or radiological method to diagnose acute appendicitis alone. Despite all advancements, it may still prove difficult to diagnose AA. This proves even more complicated in geriatric patients [15]. Advanced radiological imaging methods facilitate diagnosis. However, particularly in rural regions, surgeons may not have easy access to such advanced imaging methods [10]. Thus, studies have focused on easily accessible and cost-efficient markers with a high diagnostic value $[1,9]$. Surgeons have been interested in simple laboratory markers that can help diagnose AA and determine perforation status [16]. Complete blood count $(\mathrm{CBC})$ is an ideal marker for these properties. It can be easily accessed and quickly evaluated in many healthcare institutions, particularly in emergency rooms [10].

White blood cell (WBC) count is the most commonly used laboratory parameter for diagnosing AA. WBC count often increases in acute appendicitis patients, but it is not a specific marker because it can also increase in many other diseases [16]. One study reports that WBC has a cut-off value of 10.6, AUC: 0.66, a sensitivity of $71.2 \%$, and a specificity of $68.2 \%$ for determining perforation in the elderly [9]. Here, WBC count was found to be a marker with high sensitivity and low specificity for diagnosing AA (Cut-off value:8.97, AUC:0.72, sensitivity $85 \%$, specificity $57 \%$ ). However, it was not found to be a biomarker with high diagnostic value for determining complicated appendicitis.

As the degree of inflammation increases, the neutrophil level increases and the relative lymphocyte count decreases. Hence, the neutrophil to lymphocyte ratio (NLR) increases [17]. NLR has been used as a biomarker for morbidity, mortality, and survival in many disorders, including inflammatory and neoplastic diseases $[16,18]$. NLR has been demonstrated to be superior to other traditional infection markers like WBC, neutrophil, and CRP for determining AA severity $[9,19]$. Here, neutrophil and NLR levels were determined to be biomarkers for diagnosing AA with similar diagnostic values, while only NLR was a significant biomarker for determining complicated appendicitis.

MPV is part of routine CBC tests. It is a marker for platelet size and function. MPV has been examined for its diagnostic and prognostic values in a number of inflammatory diseases and appendicitis [20]. However, there are conflicting findings in the literature, some showing increased MPV in AA patients [21], while others showing decreased MPV [20,22]. Similar to the literature, we obtained conflicting results on MPV. MPV was the highest in the complicated appendicitis group. While MPV was expected to be the lowest in the negative appendectomy group, it was the lowest in the uncomplicated appendicitis group. Despite the 
conflicting findings, MPV was determined as a marker with the highest diagnostic value for appendicitis.

PLR is another inflammatory marker that can easily be obtained during simple hemogram tests. In many cancers and inflammatory events, the release of proinflammatory cytokines causes proliferation of megakaryocytes. Also, platelets are known to affect infections. Therefore, PLR levels can be used for diagnosing appendicitis [23]. Yıldırım et al. found PLR to be a useful marker for differentiating between complicated and uncomplicated appendicitis (cut-off value: 169.7, sensitivity: $74.4 \%$, specificity: $73.5 \%$ ) [24]. Our findings revealed that PLR was an important marker for differentiating between complicated and uncomplicated appendicitis. Although it was found to have the lowest sensitivity, it had the highest specificity (Cut off value:190.6, AUC:0.64, sensitivity 50\%, specificity $82 \%$ ).

RDW (red cell distribution width) is a subparameter that demonstrates the distribution volume of circulating erythrocytes. Inflammation disrupts blood cell maturation, damaging red cell membrane and leading to increased RDW [22]. RDW has been shown to increase significantly in complicated appendicitis, but its diagnostic values have not been specified [25]. Comparing those with appendicitis and those without, no significant difference has been found $[22,25]$. Similarly, in the current study, RDW was found to be a marker for differentiating between complicated and uncomplicated appendicitis with a high diagnostic value, but not a significant marker for diagnosing AA. (Cut off value:13.15, AUC:0.63, sensitivity 69\%, specificity 60\%).

In systemic infection cases, some bacteria, particularly E. coli, cause hemolysis and endotoxemia. Endotoxemia and bacteria result in impaired bilirubin excretion from the bile canaliculi. This leads to increased bilirubin levels in the blood [26]. It is well known that bilirubin levels increase in AA. Direct and total bilirubin levels increase in acute and complex appendicitis and are used as a diagnostic marker [27]. Despite limited in number, research has shown hyperbilirubinemia to be a biomarker for predicting perforation in geriatric patients [9]. Here, both direct bilirubin and total bilirubin levels were found to be important biomarkers for diagnosing acute appendicitis and predicting complications. Also, they were the markers with the highest specificity for predicting AA status.

The most frequently used serological indicators for diagnosing acute appendicitis are leukocyte counts and C-reactive protein (CRP) levels. CRP is an acute-phase reactant that is synthesized in the liver in response to infection or inflammation [28]. Jung et al. found CRP as a marker for determining perforation in geriatric patients with a high diagnostic value and a cut-off value of $2.09 / \mathrm{mg} / \mathrm{dl}$ [9]. Another research emphasized its high diagnostic value for determining perforation in elderly patients (AUC: 0.811 with a cut-off value of $10.19 \mathrm{mg} / \mathrm{dl})$ [29].

The most recent SIFIPAC/WSES/SICG/SIMEU guidelines recommend the use of CRP and leukocyte levels together for diagnosing AA in the elderly [30].

In determining perforation, $\mathrm{CRP}$ and $\mathrm{TB}$ are noted to be significant markers with high diagnostic value when used together [31]. In the present study, CRP was found to be an important marker for diagnosing acute appendicitis and determining complication status. Again, similar to the findings in the literature, TB and CRP were found to increase in parallel to each other (complicated appendicitis: Cut off value:5.11, AUC:0.83, sensitivity $72 \%$, specificity $76 \%$ ).

To the best of our knowledge, this is the first study in the literature to demonstrate the diagnostic efficiency of MPV, PLR, and RDW for determining acute appendicitis severity in the elderly. We can define these biomarkers as new diagnostic markers for geriatric age patients.

USG and CT are some of the basic imaging modalities that are most commonly used for diagnosing AA and determining complications. Yet, these imaging methods can be difficult to access, leading to further problems. CT has certain disadvantages, such as high costs, ionizing radiation, and contrast agent reactions. USG, on the other hand, has significant disadvantages such as being dependent on the person performing it and that it cannot be retrospectively examined $[32,33]$. Also, CT has a lower sensitivity in the elderly compared to the general population [34]. CT has been reported to have a low sensitivity for detecting perforated appendicitis without abscess or phlegm [35]. In the current study, we found CT to have a sensitivity of $77.7 \%$ and a 
specificity of $70 \%$, while USG had a sensitivity of $74.2 \%$ and a specificity of $18.2 \%$. USG has a particularly low specificity.

It is worth mentioning that the length of hospital stay was higher in the elderly compared to younger patients. Higher complication rates, prolonged antibiotic treatments, and other comorbid diseases may be associated with this finding [36]. Omari et al. found a mean length of hospital stay of 4.2 days for uncomplicated appendicitis patients and 7.4 days for complicated appendicitis patients [3]. In our patients, as expected, the longest length of stay was observed in the complicated appendicitis group, while the shortest length of stay was observed in the negative appendectomy group. With a descending order, length of hospital stay was $6.59,3.34$, and 3.13 days in our groups.

Prognosis for uncomplicated appendicitis is almost similar between young and elderly patients. However, in the case of perforation, morbidity and mortality increase dramatically in the elderly $[8,29]$. Elderly appendicitis patients have a postoperative complication rate of $21-60 \%$ and a mortality rate of $0.97-3 \%$ $[3,6,7]$. In our study, the rate of postoperative complications was $35.7 \%$, with no mortality. The rate of complications was found to be higher in the perforated patient group. The low mortality and morbidity rates in our findings can be explained by the low number of perforated appendicitis cases.

The major limitation of our study is that it is a retrospective study. Another limitation is a lack of analysis of symptoms and physical examination findings, which are crucial for diagnosing AA. Still, our research had certain strengths, including the high number of patients compared to most studies in the literature, the analyses of many biomarkers and obtaining new data, and providing more information by dividing the patients into three groups.

\section{CONCLUSIONS}

Elderly patients with abdominal pain present to hospitals later due to the obscurity of their symptoms and signs. The high rates of comorbidities in the elderly also lead to more complicated appendicitis. This results in an increased rate of postoperative complications and longer hospital stay. The USG and CT modalities used for diagnosis have almost the same, or sometimes even lower sensitivity and specificity values compared to the laboratory parameters examined here. Preoperative WBC, neutrophil, NLR, MPV, CRP, and direct and total bilirubin levels can be used in the diagnosis of AA in elderly patients. Again, NLR, PLR, RDW, $\mathrm{CRP}$, and direct and total bilirubin levels can be used to determine the complications in appendicitis.

\section{DISCLOSURES}

The authors declare that there is no conflict of interest.

\section{DATA AVAILABILITY STATEMENT}

The data that support the findings of this study are available on request from the corresponding author.

\section{AUTHORS CONTRIBUTIONS}

Conceived and designed the clinical trial: MY, RP. Performed the experiments: MY, RP. Analysed the data: MY, RP. Wrote the paper: MY, RP. Conception of the manuscript: MY, RP. Critical revisions: MY, RP.

FUNDING: No funding.

\section{Hosted file}

Tables.pdf available at https://authorea.com/users/410035/articles/519536-the-role-of-basiclaboratory-parameters-in-diagnosing-acute-appendicitis-and-determining-disease-severityin-the-elderly

\section{Hosted file}


figures.pdf available at https://authorea.com/users/410035/articles/519536-the-role-ofbasic-laboratory-parameters-in-diagnosing-acute-appendicitis-and-determining-diseaseseverity-in-the-elderly 